\title{
Wind Turbine Mounted on A Motorcycle for Portable Charger
}

\author{
G. Subhashini, Raed Abdulla, Thinaga Raj R Mohan \\ School of Engineering, Asia Pacific University of Technology and Innovation (APU), Technology Park Malaysia, \\ Bukit Jalil, Kuala Lumpur, Malaysia
}

\begin{tabular}{l}
\hline \hline Article Info \\
\hline Article history: \\
Received Dec 27, 2017 \\
Revised Jul 14, 2018 \\
Accepted Sep 18, 2018 \\
\hline
\end{tabular}

Keyword:

Arduino microcontroller

Portable charger

Wind energy

Wind turbine

\begin{abstract}
This paper aims to develop a wind energy harvesting system to charger an electronic device of $5 \mathrm{~V}$ during traveling period from a place to another. Upon the situation faced by the two wheeler depending on the conventional power socket, this system was constructed to overcome the dependencies of power since the natural energy can be harvested to produce an electrical energy. The power storage unit system was done by integrating supercapacitor to storage the electrical energy hence an arduino microcontroller was been integrated with supercapacitor which able to display the percentage and voltage stored in the supercapacitor. The maximum speed of the geared DC generator is $1297 \mathrm{rpm}$ and based on the testing carried out, it was observed that the maximum voltage produce at $1297 \mathrm{rpm}$ able to produce $17 \mathrm{~V}$ which sufficient to power up the entire system as it required $5.3 \mathrm{~V}$ for operating. Besides, the system was converted into acrylic board since the system are to be implemented on two wheeler hence the acrylic board was design based on the conform ability of the user. In a nut shell, the functionality of the entire system was successfully achieved based on the objectives and overcoming the drawback from past research work.
\end{abstract}

Copyright () 2018 Institute of Advanced Engineering and Science. All rights reserved.

\section{Corresponding Author:}

Raed Abdulla,

School of Engineering,

Asia Pacific University of Technology and Innovation (APU),

Technology Park Malaysia, Bukit Jalil, Kuala Lumpur 57000, Malaysia.

Email: dr.raed@apu.edu.my

\section{INTRODUCTION}

In the recent days, with rapidly increasing population, natural resources are being exploited to its maximum. Some studies has stated that, availability of oil will vanish in another 15 decades and the global energy demand will increase three times by the year of 2050. Considering the impact for the future generation, numerous studies has been carried out emphasizing on sustainable development and efficient energy conservation. Many inventions has been made to utilize the renewable energy resources for various applications [1]. The two important factors regarding renewable energy are energy management and saving energy [2]. Most commonly harnessed renewable energy are wind, hydro and sunlight. Among the three gifts of mother-nature, power produced from wind are known to be reliable energy because anything which moves produces wind besides naturally generated wind. Moreover wind energy does not cause greenhouse effect during the process of conversion from mechanical to electrical energy.

Wind energy is the energy present in moving air. Past studies has been focused on harnessing the wind energy for large scale applications such as to produce electricity for rural areas, running a windmill and pumping water from a well. Recent studies on the other hand are being focused on harvesting wind energy for smaller scale portable applications. In such application, smaller turbines are designed in such manner where it can be mounted on any type of land vehicle with adequate storage capacity to power up most of the electronic devices available in the market. 
A portable charger containing power storage, also known as "Power Bank" is a device that has been a source of charging medium during the absence of power supply and being compatible with most electronic gadgets. Portable storage bank contains rechargeable cells which are able to store an adequate amount of charge which is sufficient enough to charge a device fully at least twice. In recent days where electronic gadgets are one of the most important aspect in human's life as a medium of communication, it becomes very difficult to charge a device while travelling from one place to another due to availability of power supply and especially when the power bank is fully drained. Wind energy produced from moving modes of transportation are mostly being wasted for an instance, wind produced from a passing by train, car and motorcycle.

According to [3], the author justified that considering the worldwide crisis and depletion of mineral resources due to over exploitation, this will lead to shortage in generation of power which is an issue being faced in most of the countries with large population. Thus, the problem targeted to be solved by the author was to implement a sustainable solution to provide electricity in order to charge mobile phones and laptops. Elaborating the method implemented in this research, the mechanism contained a single power extraction medium which is wind energy. The design implemented for the turbine was a vertical axis blade instead of the traditional blades. The blades were held by an 8 inch cylindrical rod made of PVC which is light weight besides being rigid. Two types of blades were assembled onto the rod where one being the turbine with larger surface on the inner part and the other one having a bendable smaller surface on the outer part.

Another study conducted by [4] to overcome the problem where people are unable to charge their mobile phones while travelling from one place to another. The system was basically a hybrid charger where the mobile phone can be charged with a $220 \mathrm{~V}$ power supply, a wind harvesting system to produce power and a manually human powered power generator. The authors implemented a DC generator incorporated with a turbine which rotates with presence of air for wind energy harvesting. The $220 \mathrm{~V}$ charging mechanism on the other hand consists of a step down transformer which converts $220 \mathrm{~V}$ AC into $9 \mathrm{~V} \mathrm{AC}$ and further converted to DC using a bridge rectifier which produces $5 \mathrm{~V}$ DC.

To charge battery, charger is an essential device used to charge a secondary cell, rechargeable battery by forcing an electric current through it [5]. Similarly, the authors in [6] carried out a research on developing a system to charge the battery of an electric motorcycle. Apart from being cost effective, the electrically driven two-wheeler does not produce carbon emission. The existing electric motorcycle are fully relying on the power source available at home or charging stations to be charged. Thus, while travelling a distance where the battery is about to be fully drained, the user has to locate the nearest charging station to charge the vehicle sufficient enough to continue their journey. Thus, the system implemented by the author comes in handy, where while the two-wheeler is on motion, the battery can be charged by harvesting the wind energy available from the surrounding.

The wind turbine plays as a prime mover to a connected DC generator [7]. A different approached was carried out in [8], author focusing on implementing renewable energy by combining wind and solar energy harvesting system for continuous electric vehicle battery charging. The author has concentrated on this approach due to distress caused such as the global warming, environmental pollution and oil price increases which are common problems faced worldwide. Moreover, approximately 60 to 90 percent of air pollution is subjected to heavy usage of vehicles in urban areas and availability of oil will vanish in another 150 years. Thus, electric vehicles are one of the best solution for green economy development and also to decrease the stress of universal issues as stated by the author. Based on the analysis done, the power extraction from the solar was not as promising as the wind turbine because only about $35 \%$ of the solar irradiance was potentially available for conversion to electrical power. The overall results obtained by combining both the solar and wind energy extracted the output power was up to $2.5 \mathrm{~kW}$ during the motion of the vehicle and the power discharge was $25 \%$ for every $10 \mathrm{~kW}$ use electric motor of the vehicle.

In [9], the authors carried out a study emphasizing on energy storage system for wind turbine generators. In order to achieve an optimum performance of the energy storage system, combination of two different technologies such as Vanadium-redox flow battery (VRB) and supercapacitor integrated with the generators improves the efficiency of power generation and storage. The author has stated that, although there are wide range of storage technologies, but unfortunately the specifications does not fulfill the requirements of microgrid application. Microgrid is used to manage interconnected loads and distributed energy sources. It can be configured independently as well as parallel with the existing utility grid system. The model is based on a supercapacitor holding the characteristic of $0.58-\mathrm{F} 400-\mathrm{V}$.

Research carried out by [10], for a portable wind mill to charge mobile phones consists of a legion meter instead of a storage. The wind turbine was connected to electric generator which is able to produce high output at lower RPM. Upon testing, the electric generator was only able to produce an output of 0.4 to 0.5 amps which is not sufficient to charge any device. Thus, the author integrated a legion meter to boost the output produced by the electric generator. A legion meter has a voltage range of 3 to $6 \mathrm{~V}$ and power range up 
to 22.2 watts. The circuitry of a legion meter consists of boost converter which is also known as an amplifier which amplifies the input by five times. Based on the results, the gain produced by the legion meter varied for different types of hand phones. This is due to the power rectifier installed in the legion meter which limits the amplification based on the required output. This technology prevents excessive power which prevents damage to the device connected. The gain produced by the legion meter ranged from 10 up to $92 \%$.

As indicated by [11], utilization of non-conventional energy sources and its latest research is one of the essential analysis to be carried to benefit the people and industries. Many heavy machineries tend to produce ample of energy while operating which are being wasted and if those energy are properly utilized, it can be used to generate power. Since early days, wind energy is one of the alternate source of energy being utilized by the society for the purpose of development. In view of the technique actualized by [8], the turbine designed depended on the property of DC motor that when the shaft of a DC motor beings to rotate, it is able to produce voltage similar to a generator. This model utilized three DC motors which were associated with turbine blades to ensure rotational movement during presence of wind energy. The turbine was built utilizing aluminum pieces of width $7 \mathrm{~mm}$ and thickness $2 \mathrm{~mm}$.

Another investigation done by [12], the authors expressed that the Agile Wind Energy Harvesting Technique is fundamentally the era of milliwatt electrical power from the flow of wind energy is generally new contrasted with the ordinary power generating methods. The mediums used to harvest the wind energy consists of settled segments and dynamic parts, which are vibrated by mechanical energy produced by wind. The amount of power generated relies upon the size of the system, velocity of the wind energy and surrounding condition. As indicated by the author, Albert Betz inferred that a wind turbine hypothetically is unable change over $59 \%$ of the kinetic energy produced by wind to mechanical energy whiich is known as Betz confinement.

The main objective of this proposed research is to design and develop a wind energy harvesting system to charge a power bank on a motorcycle. The proposed system is expected to deliver an output of $5 \mathrm{~V}$ which is sufficient to charge any portable storage bank. Moreover, when there is no need of charging, the system must be able to store the generated power which can be utilized anytime.

Furthermore implementation of a power level monitoring is to ensure the user is able to view the percentage of charge used up without the need of grabbing the storage unit to see how much has the portable storage bank charged. This guarantees the safety of the motorcyclist is by avoiding distraction. Most importantly, the system is created focusing on the elements of sustainability.

Moreover, the proposed system is expected to operate under outdoor condition since it will be implemented on a motorcycle. Hence, the proposed system is able to overcome the problem of needing a power source to charge any $5 \mathrm{~V}$ electronic devices while travelling one place to another. Last but not least, the proposed system is user friendly which does not require a special skilled individual to operate or monitor.

\section{RESEARCH METHOD}

The proposed methodology consist of two major parts. Since the research is mainly emphasizing on developing a wind energy harvesting system for a portable charger, the very first element based on the block diagram is the DC generator which produces the electrical energy. The second element which is also the enhancement part included in the system is the power storage unit having the ability supply sufficient power to the Arduino microcontroller and charging the power bank simultaneously.

A wind turbine is attached to the DC generator by implementing the coupling method using an iron shaft as the medium. The rotation of the turbine is based upon the volume of air being applied on the blades. When the shaft connected to the DC generator begins to rotate, conversion of a mechanical to electrical energy occurs in the DC generator. The converted electrical energy then passes through the Buck converter to produce a stable $5 \mathrm{~V}$. Supercapacitor is used as the power storage unit which plays the role to store and discharge the power upon connecting a load.

As illustrated in Figure 1, the operation of the entire system initializes when the wind turbine starts rotating with presence of wind energy. When optimum RPM is attained, this scenario enables the DC generator to produce electrical energy which charges the supercapacitor. Meanwhile, the Schottky Diode ensures the flow of electrical energy in one direction. Moving on to the decision box, in the case of supercapacitor being fully charged, it is now ready to discharge whereas, if it is not being fully charged, it continues charging till it reaches the maximum capacity and stops charging. The reason supercapacitor being chosen as the storage unit is because it has the ability to charge within a small period of time and discharge gradually unlike other storage units. Since the Arduino needs a steady $5 \mathrm{~V}$ to operate efficiently, the stored electrical energy is being pass through a Buck Converter. This is due the configuration of the supercapacitor placed in parallel to increase the amount of storage capacity. 


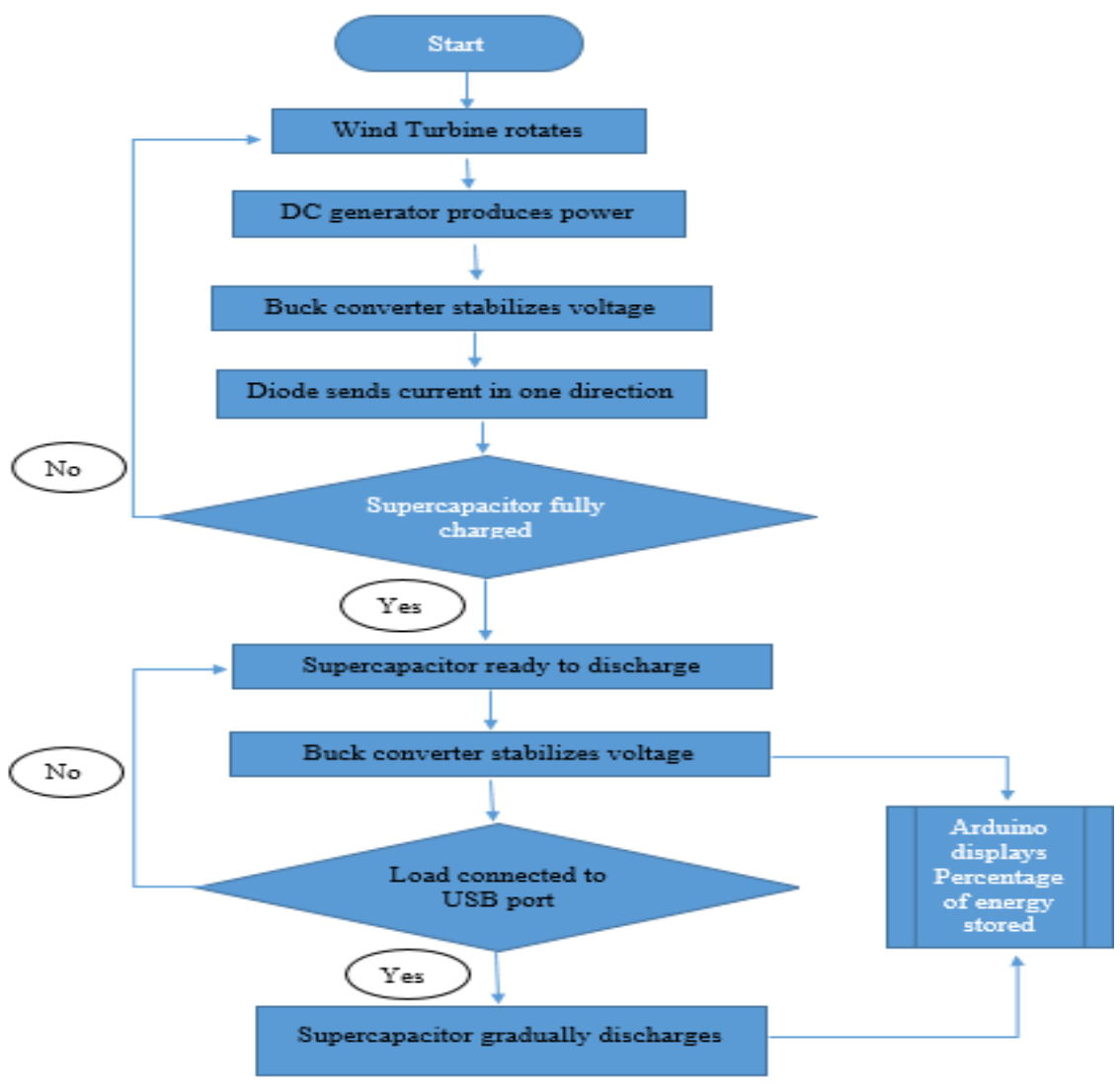

Figure 1. Flow chart for wind energy harvesting for portable charger

Thus, when all the supercapacitors discharges simultaneously, the amount of voltage produced will be higher than $5 \mathrm{~V}$. Moreover, this ensures the Arduino is not overloaded where it eventually heats up and shuts down. The Arduino then initializes to display the amount of energy stored in the supercapacitor. This process takes place only while charging the supercapacitor for the very first time where there is completely no charge in the supercapacitor. For the following cycles, the Arduino stays ON as long as there is sufficient amount of storage in the supercapacitor. Besides initializing the Arduino, a connection is given to a 5V USB female port where the power bank acting as the load is able to charge from the energy stored in supercapacitor. The supercapacitor only starts discharging gradually with a steady $5 \mathrm{~V}$ stepped down from the buck converter when there is a load being connected.

\section{SYSTEM IMPLEMENTATION}

Initially, based on the proposed methodology, the diode was placed at the output of the buck converter. This was to ensure the voltage harvested from the DC generator flows in one direction to improve the efficiency of charging. The output of the buck converter was set at $5.3 \mathrm{~V}$ since the supercapacitor implemented to store the charge was rated at $5.4 \mathrm{~V}$ when connected in series. The diode on the other hand has the characteristics of internal resistance. [11] Thus, when it was placed at the output of the buck converter the voltage was further stepped down which was not sufficient to charge the supercapacitor efficiently.

Since the DC generator is able to produce an average of $20 \mathrm{~V}$, the diode was then placed at the output of the DC generator and the input of the buck converter. Upon testing, it was found that the buck converter was able to produce an output of $5.3 \mathrm{~V}$ which was sufficient enough to charge the supercapacitor efficiently. Another modification carried out was by implementing two different booster modules with different specifications to power up the Arduino and to charge the load individually. This was to ensure the efficiency of the supercapacitor to produce sufficient charge to both the Arduino and load.

Besides, two switches were integrated at the output of the supercapacitor respectively. One switch is responsible to turn the booster module connected to the Arduino ON and OFF in order to display the 
percentage of charge left in the supercapacitor and the other switch is responsible for turning on the 5V USB booster module to charge the load.

The switch plays an important role as a safety feature since it enables the user to turn OFF supply to the load when it is fully charged. Hence, the user does not need to unplug the device while cruising on the motorcycle if the device is fully charged which avoids distraction in other words. Moreover, the Arduino was initially powered via the $5 \mathrm{~V}$ and ground pin connected directly to the output of the supercapacitor rated at $5.4 \mathrm{~V}$ and resulted in excessive heating on the Arduino microcontroller board.

The modification was carried out by implementing the 5V USB booster which is able produce sufficient $5 \mathrm{~V}$ with minimal input of $0.9 \mathrm{~V}$. A USB connecter cable was then used to integrate the female USB with Arduino. The final modification which has been carried out was on the software part of the system where an additional coding was generated in order to display the percentage of voltage as well as the voltage divider equation since a voltage sensor was implemented in order for the microcontroller to read voltage above 5V. Figure 2 illustrates construction of the overall mechanism on a breadboard.

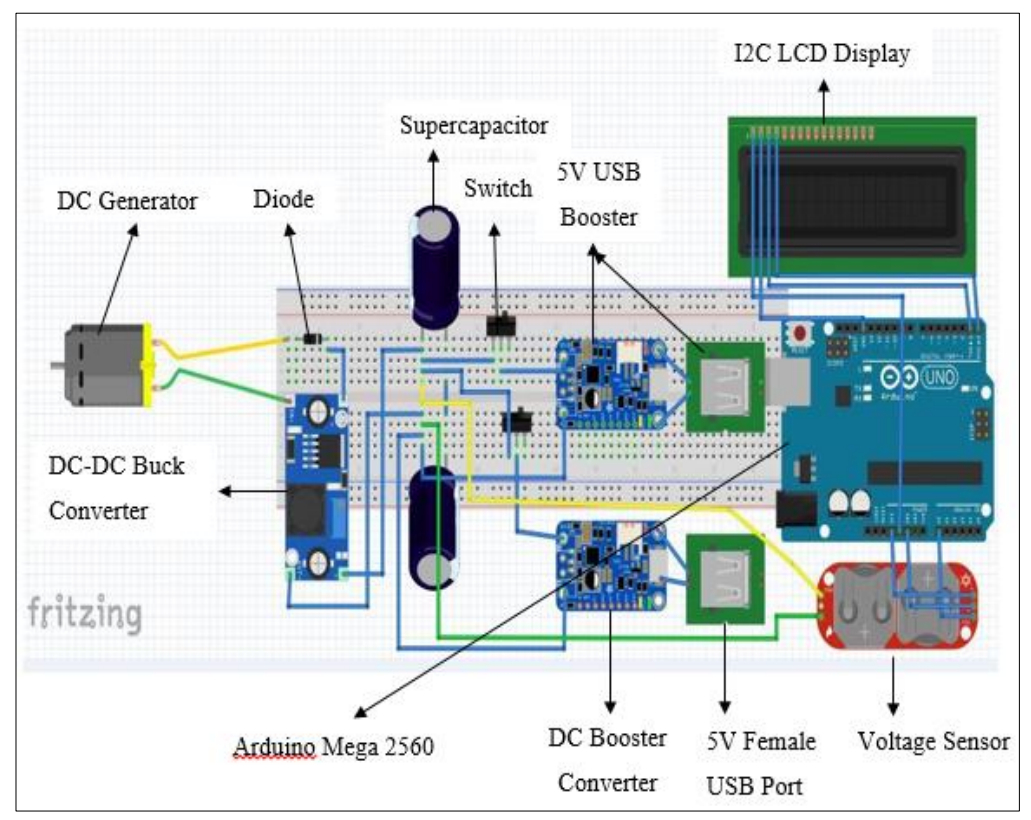

Figure 2. Prototype configuration on breadboard

\section{RESULTS AND ANALYSIS}

\subsection{Test for Types of DC Generator}

The four samples of blade count were of two, three, four and five blades respectively. The test was first carried out on the geared DC generator by integrating the wind turbine with two blades using a coupler. Upon fitment, the wind turbine was manually spin to ensure it is locked in place and has a balance rotary movement as a safety measure. Secondly, two multicore wires were attached to the positive and negative terminal of the DC generator to extract the voltage produced from its rotation which were directly connected to the probes of the multimeter. Two different graphs were generated to analyze the maximum voltage and rotational per minute produced by the geared DC generator and PMSG DC generator respectively. Analyzing the graph in Figure 3 which represents the geared DC generator, it can be seen that the maximum voltage produced is increasing proportionally as the number of blades increases followed by the maximum rotational per minute.

Referring to Figure 4 representing the graph generated for the PMSG DC generator, the characteristics of voltage produced alongside the number of blades were similar as the previous generator. Despite, there were several differences in the maximum voltage and RPM produced. The geared generator was able to produce a maximum of 17 volts with RPM rated at 1297 whereas the PMSG was only able to produce a maximum of 12 volts at RPM rated at 2900. Hence, comparing both the outcomes, the geared generator is found to be more suitable to be implemented in this system since higher voltage can be obtained at a lower RPM rate which is considered efficient. 


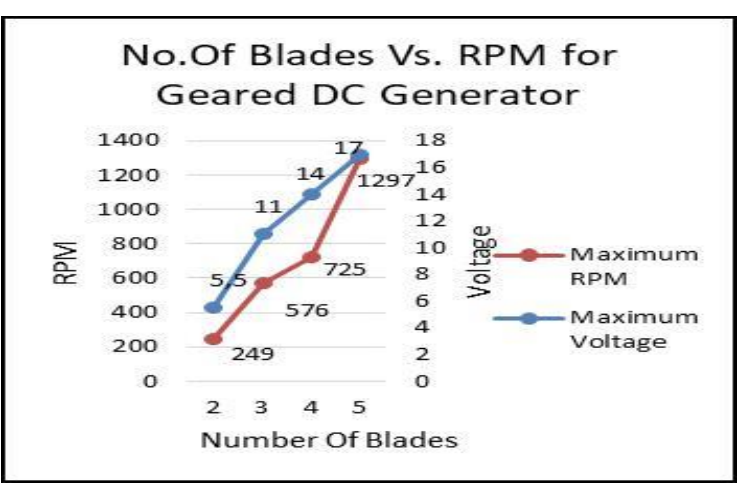

Figure 3. Graph for number of blades against RPM for PMSG

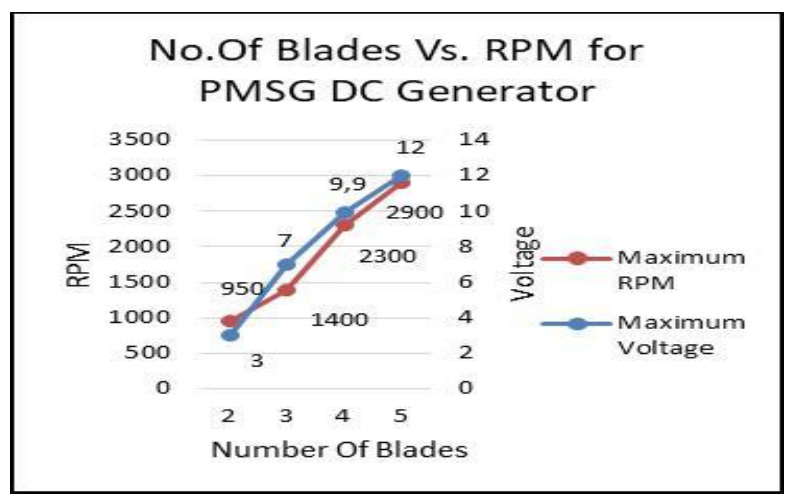

Figure 4. Graph for Number of blades RPM of geared DC generator

\subsection{Test for Dimension of Turbine}

Three different wind turbines with three different sizes respectively were first placed on a graph paper and shapes of turbines were drawn on it. The surface area for each turbine was then calculated based on the standard dimension of the graph paper where 5 boxes represents one inch and noted on the table. Following the similar procedure from the previous test, all the wind turbines were coupled to the DC generator one after another and safety measures were emphasized upon fitment of each turbine. The motorcycle was ridden at a maximum speed of $100 \mathrm{~km} / \mathrm{h}$ and the voltage produced for three different surface areas of turbines with four different number of blades for each size were recorded. The voltage reading were taken from the digital multimeter connected to the wires attached to the positive and negative terminals of the generator.

A chart was generated as shown in Figure 5 with each series representing different sizes of turbine as measured in the beginning of the test. Each bar with different colors represents different number of blades as described in the legend included in the chart. Based on the obtained results, the maximum voltage produced by the three different sizes of turbine with four different number of blades each had a difference of approximately 3 volts. The turbine with the smallest surface area was able to produce the highest voltage for all four different number of blades which has proven the theoretical theory.

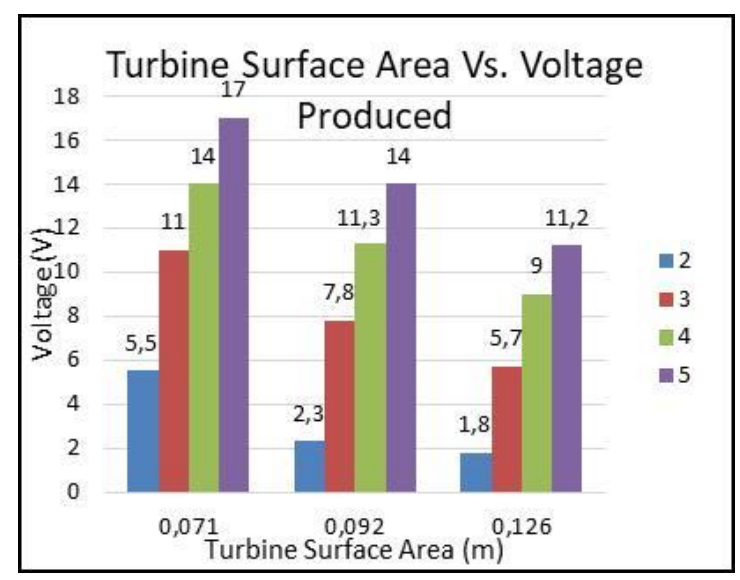

Figure 5. Graph for surface area of turbine against voltage produced

\subsection{Test for Wind Speed}

Using the same system configuration and the surface area of turbine selected from the previous test, this experiment was began by first identifying the lowest wind speed required to drive the turbine with five blades since it is the maximum number of blades used for all the testing carried out. The wind speed was identified based on the speed of the motorcycle rated in $\mathrm{km} / \mathrm{h}$ which was then converted to $\mathrm{m} / \mathrm{s}$. 
The motorcycle was gradually accelerated from $0 \mathrm{~km} / \mathrm{h}$ and the wind speed was tabulated at the point where the turbine began to rotate followed by the voltage produced which was displayed on the multimeter. Based on the tabulated data, a graph were generated with each line marker representing different number of blades ranging from two to five blades as illustrated in Figure 6.

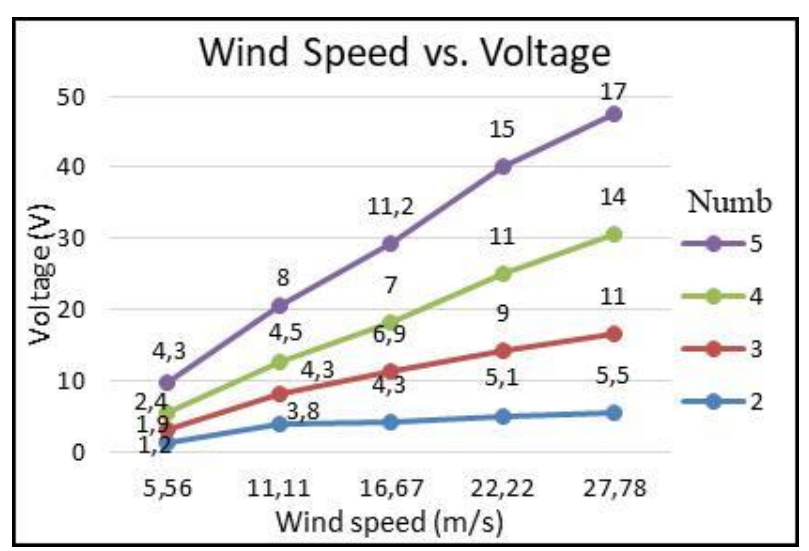

Figure 6. Graph for wind speed against voltage produced

\subsection{Test for Number of Blades}

Upon identifying the most suitable surface area of turbine to be used and voltage produced by each turbine with different number of blades at various wind speed, the following test was conducted to identify the most suitable number of blades to be implemented using the fixed surface area of turbine which was 10.4 by $10.4 \mathrm{~cm}$ for each blade.

Theoretically, based on the calculation made in the previous chapter, the more number of blades enables lesser drag force on the surface of turbine enabling it to spin faster. Thus, the theory was put to test using four different number of blades with the same surface area. The motorcycle was the ridden at two different speed which was 50 and $100 \mathrm{~km} / \mathrm{h}$ producing wind speed rated at 13.89 and $27.78 \mathrm{~m} / \mathrm{s}$ respectively. The reason behind testing it under two different speed of motorcycle is to apply the real time scenario since the motorcycles are not always ridden at the maximum speed and it varies among users and road condition hence two practical speed were elected for this test.The test was carried out by remaining the same experimental configuration as the previous experiment and obeying to crucial safety measures while assembling the wind turbine for every sample. The motor was first ridden at $50 \mathrm{~km} / \mathrm{h}$ being able to produce a wind speed of $13.89 \mathrm{~m} / \mathrm{s}$ and the voltage and RPM produced were measured using digital multimeter and photo type tachometer followed by tabulation of data. Upon completing the same procedure for all the four turbine samples, the data for the voltage and RPM produced at $100 \mathrm{~km} / \mathrm{h}$ of motorcycle speed was obtained from the data tabulated during the first experiment. Table 1 illustrates four different number of blades tested at two different wind speed with relation to the rotational per minute and voltage produced.

Table 1: Test for Number of Blades

\begin{tabular}{cccc}
\hline Motorcycle speed $(\mathrm{km} / \mathrm{h})$ & No. of Blades & Rotational Per Minute & Voltage $(\mathrm{V})$ \\
\hline \multirow{3}{*}{50} & 2 & 90 & 1.7 \\
& 3 & 175 & 3.9 \\
& 4 & 290 & 7.0 \\
& 5 & 375 & 8.8 \\
\multirow{3}{*}{100} & 2 & 249 & 5.5 \\
& 3 & 576 & 11 \\
& 4 & 725 & 14 \\
& 5 & 1297 & 17 \\
\hline
\end{tabular}

\section{CONCLUSION}

The project was successfully achieved by developing a wind energy harvesting system for portable charger by integrating several components and Supercapacitor as the power storage unit. The working principle of the system was based on four objectives as derived at the beginning of this project. Several 
calculations and testing were performed to identify the most ideal turbine to be used with the system to perform at its maximum efficiency. The wind harvesting mechanism was assembled in a waterproof casing since it is to be implemented on a motorcycle. A universal bracket was constructed with the wind harvesting mechanism which gives an upper hand to the system since it can be mounted on any type of motorcycle with the aid of bolt and nut. The voltage produced from the DC generator was stepped down to 5.3V using the DC-DC buck converter. The Supercapacitors were connected in series which was able to store and produce voltage up to $5.4 \mathrm{~V}$ being sufficient to power up the Arduino microcontroller as well as to charge a powerbank simultaneously.

\section{REFERENCES}

[1] Rahaman S, Patil M. G. Development of wind powered mobile charger. International Journal of Research in Aeronautical and Mechanical Engineering. 2015; 3(11): 26-39.

[2] Meenakshi V, Paramasivam S. Control Strategy Used in DFIG and PMSG Based Wind Turbines an Overview. International Journal of Power Electronics and Drive Systems (IJPEDS). 2017; 8(3):1160-1167.

[3] Chidurala R. Electrical devices charging with portable mini wind mill. International Journal of Current Engineering and Technology. 2014; 4(4): 2831-2832.

[4] Reddy N R R, Sreekanth Y, Narayana M. Mechanical and electrical mobile charger. International Journal of Engineering Research and Applications. 2013; 3(6):1705-1708.

[5] Rakhi K, Karuppasamy I, Nair MG. Hardware Implementation of Solar Photovoltaic System based Half Bridge Series Parallel Resonant Converter for Battery Charger. International Journal of Power Electronics and Drive Systems (IJPEDS). 2017; 8(4).

[6] SINGH S N, SUMIT.Jha S K, Sinha S K. Wind driven mobile charging of automobile battery. International Journal of Engineering, Science and Technology. 2013; 3(5): 113-119.

[7] Jannati M, Eskandari S, Sutikno T. Speed Control of 3-phase Induction Motors under Fault Conditions Supplied by Wind Turbine Using Indirect Vector Control. International Journal of Power Electronics and Drive Systems (IJPEDS). 2017; 8(1): 222-30.

[8] Tekle A. Renewable energy use for continuous electric vehicles battery charging capacity in mobile. International Journal of Engineering Research and Applications, 2014; 5(10): 58-65.

[9] Spriya S, Rajakumar S. An energy storage system for wind turbine generators-battery and supercapacitor. International Journal of Engineering Research and Applications (IJERA). 2013; 3(2):1219-23.

[10] Babu NR, Arulmozhivarman P. Wind energy conversion systems-a technical review. Journal of Engineering Science and Technology. 2013; 8(4):493-507.

[11] Sengupta A, Solanki SS. Design and Modeling of a Small Scale Wind Turbine Generator. International Journal of Research in Engineering and Science. 2013; 1(5): 61-68.

[12] Nabavi S, Zhang L. Portable wind energy harvesters for Low-Power applications: A survey. Sensors. $2016 ; 16(7): 1101$.

\section{BIOGRAPHIES OF AUTHORS}

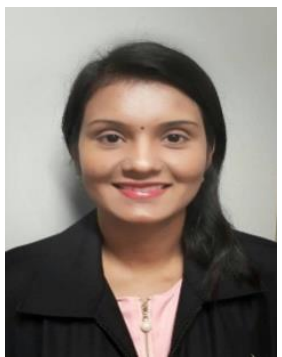

Subhashini Gopal Krishnan Subhashini Gopal Krishnan received the Bachelor's degree in Electrical and Electronics Engineering from University Tenaga Nasional, in 2009, the Master degree in Engineering Management from University Tenaga Nasional, in 2011. He is currently a Lecturer at the School of Engineering, Asia Pacific University of Technology and innovation (APU) in Bukit Jalil, Kuala Lumpur, Malaysia. He has contributed in research and in the areas of renewable energy, sustainable development and engineering management. Her research output was translated to numbers of awards and publications. She is also a Chartered Engineering in the institution of Mechanical Engineers.

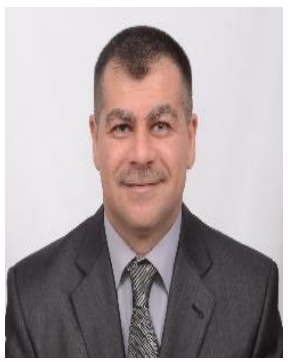

Dr. Raed Mohammed Taher Abdulla received the Bachelor's degree in Electrical engineering from AL-Mustansiria University, Baghdad, Iraq in 1997, the Master degree in Electronic Systems Design Engineering from Universiti Sains Malaysia, Malaysia in 2008 and he received his $\mathrm{PhD}$ in Wireless and Mobile Systems from Universiti Sains Malaysia, Malaysia in 2012. He is currently a Senior Lecturer at the School of Engineering, Asia Pacific University of Technology and innovation (APU) in Bukit Jalil, Kuala Lumbur, Malaysia. He has contributed in research and in the areas of Radio Frequency Identification (RFID) and Wireless Sensor Network (WSN). His research output was translated to numbers of awards and publications. He is a member in the institution of engineering and technology (IET). Dr. Raed also member in Centre of Research and Development of IoT (CREDIT), Asia Pacific University of Technology and Innovation (APU). 


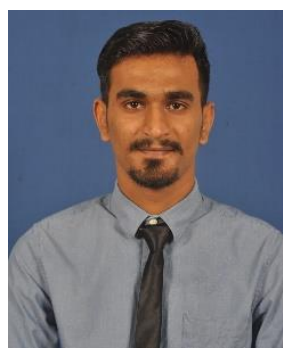

Thinaga Raj R Mohan has completed his high school studies in year of 2010 and joined Faculty of Engineering in the year of 2011. In year of 2012 started his Bachelor degree of Electrical \& Electronic Engineering and major in Electrical Engineering at Asia Pacific University and currently walk into his working career as an Electrical Engineer at Melatone Sdn Bhd one of a Korean company branch which located in Malaysia. 